\title{
Desafíos del modelo de práctica resonancia colaborativa en la formación inicial de docentes
}

\section{Desafios do modelo de prática por ressonância colaborativa na formação inicial dos docentes}

\section{Challenges of the collaborative resonance practice model in early teacher training}

\author{
Eduardo Rodríguez Zidán ${ }^{1}$ \\ Javier Grilli²
}

\begin{abstract}
RESUMEN
En este artículo se analizan y comparan los dos modelos de práctica docente para formación de profesores de educación media implementados en Uruguay: el modelo histórico, basado en un trabajo individual con escasa supervisión pedagógica, y el modelo por resonancia colaborativa. El diseño de investigación fue mixto y de tipo concurrente articulando estrategias metodológicas cualitativas y cuantitativas. Se aplicó una encuesta a una muestra no probabilística de 197 casos (122 estudiantes practicantes de profesorado y 75 profesores de centros de práctica) y entrevistas en profundidad a 13 informantes calificados. Luego de revisar y comparar diferentes estudios internacionales y del análisis de evidencias recogidas, se concluye que el modelo denominado "resonancia colaborativa" es una alternativa eficaz para orientar cambios significativos en la formación práctica de los futuros docentes de educación media. La implementación de esta modalidad implica un importante desafío de gestión institucional pero es un paso importante en
\end{abstract}

DOI: $10.1590 / 0104-4060.46406$

1 Universidad ORT Uruguay. Centro Regional de Profesores del Litoral Salto Uruguay. Montevideo, Montevideo, Uruguay. Cuareim, 1451, 11100.E-mail: cerzidan@yahoo.com.ar

2 Administración Nacional de Educación Pública. Centro Regional de Profesores del Litoral. Salto. Uruguay. Calle Florencio Sánchez, 398.E-mail: javigrill@hotmail.com 
el largo camino de fortalecimiento de la calidad en la formación de profesores para la educación media.

Palabras-clave: formación de profesores; práctica docente; resonancia colaborativa.

\title{
RESUMO
}

Neste artigo são analisados e comparados dois modelos de prática docente para a formação de professores do ensino médio implementados no Uruguai: o modelo histórico, baseado em um trabalho individual, com pouca supervisão pedagógica, e o modelo por ressonância colaborativa. O método de investigação foi misto e do tipo concorrente, articulando estratégias metodológicas qualitativas e quantitativas. Foi aplicada uma entrevista a uma amostra não probabilística de 197 casos (122 estudantes praticantes da carreira docente e 75 professores de centros de práticas), bem como entrevistas em profundidade com 13 informantes qualificados. Depois de revisar e comparar diferentes estudos intencionais e da realização da análise das evidências coletadas, foi concluído que o modelo denominado "ressonância colaborativa" é uma alternativa eficaz para orientar mudanças significativas na formação de futuros docentes do ensino médio. A implementação desta modalidade é um importante desafio de gestão institucional e é um passo importante no longo caminho para o fortalecimento da qualidade da formação de professores do ensino médio.

Palavras-chave: formação de professores; prática docente; ressonância colaborativa.

\begin{abstract}
This article analyzes and compares two models of teaching practice for the training of secondary education teachers implemented in Uruguay: the historical model, based on individual work with little pedagogical supervision, and the model for collaborative resonance. The research design is opposing and mixed, articulating qualitative and quantitative methodological strategies. A survey was applied to a sample of 197 cases with non probability (122 practitioner students of the teaching career and 75 teachers of practice centers) and in-depth interviews with 13 qualified informants. After reviewing and comparing various international studies and the analysis of collected evidence, we concluded that the model called "collaborative resonance" is an effective way to guide significant changes in the practical training of secondary education future teachers. The implementation of this approach involves an important challenge for institutional management and it is an important step in the long way to strengthen the quality of teacher training for secondary education.
\end{abstract}

Keywords: teacher training; teaching practice; collaborative resonance. 


\section{Introducción}

Este documento presenta un avance de los resultados de una investigación sobre las características y los procesos de formación de profesores de educación media; se trabajó analizando las diferentes experiencias de práctica profesional en la etapa de formación inicial, de los futuros docentes de educación secundaria en Uruguay ${ }^{3}$.

En el país existen dos modelos de formación práctica de profesores para la enseñanza media. El más desarrollado (desde el año 1951), es la práctica docente de tipo individual: los estudiantes de profesorado, denominados en Uruguay practicantes (de $2^{\circ}, 3$ er año y 4 to año de la carrera), asisten a varios establecimientos e instituciones de nivel medio para realizar su práctica docente.

De esta manera los practicantes suelen vivir la práctica como un momento separado de la instancia de formación teórica, en distintos centros educativos de enseñanza media, con baja coordinación y muy poca comunicación con otros estudiantes practicantes (de la misma u otra especialidad) ${ }^{4}$. Esta modalidad de aprendizaje del rol docente se hace en soledad ya que los estudiantes de profesorado representan un número ínfimo en el total de docentes que se desempeñan en la Escuela secundaria que los acoge.

Este primer modelo de formación práctica, de fuerte tradición en Uruguay, se basa en un trabajo individual del practicante donde la supervisión pedagógica del profesor de didáctica del centro de formación inicial es escasa. Un segundo modelo alternativo es el promovido a partir de la reforma curricular del año 1997. Se introduce aquí como innovación la práctica docente basada en la colaboración entre los centros educativos y el desarrollo de dispositivos de apoyo a la formación como el dispositivo "pareja pedagógica".

En este segundo modelo, el establecimiento de educación media - que denominaremos en este artículo Escuela de práctica ${ }^{5}$ - es concebido desde la

3 "Modelos de práctica docente en Uruguay", proyecto aprobado para su realización por la dirección del Centro Regional de Profesores del Litoral, 2012-2014.

4 Especialidad: formación académica del profesor que generalmente se identifica con la disciplina o campo del saber vinculado a su desempeño profesional. El Sistema Único Nacional de Formación Docente (SUNFD, 2008), vigente en la actualidad, identifica más de 20 especialidades de profesorado, como por ejemplo: Ciencias Biológicas, Comunicación Visual - Dibujo, Física, Química, Matemática, Idioma Español o Educación Musical.

5 En América Latina son múltiples las formas de denominación del nivel medio o secundario. En este artículo asumimos la designación de Educación Media (básica o superior) apoyándonos en los establecido por la Ley General del Educación 18.347 de Uruguay. Para una discusión más 
teoría como un espacio de formación con una fuerte coordinación de las prácticas pedagógicas y de supervisión, la evaluación y el trabajo en proyectos de coordinación docente con la institución de formación inicial.

El foco de este artículo es analizar la modalidad basada en acuerdos de trabajo con la Escuela de práctica que promueven, por este motivo, una fuerte concentración de profesores practicantes en un determinado centro educativo medio.

Las preguntas centrales que guiaron nuestra investigación fueron las siguientes: ¿cómo valoran los estudiantes de profesorado, docentes de secundaria, profesores formadores de docentes y otros actores de la enseñanza, la experiencia de formación en un centro de educación media basada en proyectos de colaboración con el centro de formación inicial?; ¿cuáles aspectos son considerados fortalezas y cuáles son considerados debilidades en la nueva modalidad de formación?; ¿existen diferencias significativas en las opiniones de los diferentes colectivos consultados sobre esta modalidad de práctica docente?

\section{¿Qué dicen la evidencia y los estudios internacionales sobre los modelos de práctica?}

No existen dudas de que el núcleo práctica docente, y la relación entre la teoría y la práctica profesional, es uno de los ejes fundamentales en la formación de profesores. La articulación entre el Centro formador de docente y la Escuela de práctica es especialmente importante para que en la práctica docente se establezca una buena relación con la teoría pedagógica.

La literatura académica y diversos estudios en la materia orientados al análisis del proceso de articulación entre los centros de formación inicial y los centros de inserción laboral de los estudiantes en formación señalan las dificultades que se deben superar para lograr una experiencia de formación que no reproduzca hábitos tradicionales y modelos acríticos de la profesión docente. En tal sentido, la construcción del conocimiento profesional en los futuros docentes debe comenzar en la etapa de formación y a través de dispositivos que promuevan la reflexión sistemática y la construcción del rol. Sanjurjo (2012, p. 2), entre otros dispositivos de formación, destaca los siguientes: la residencia 
anual ${ }^{6}$, la inserción institucional (no exclusivamente áulica o al proyecto), la relación prolongada de la cátedra con determinados tutores e instituciones, la delimitación de un "mapa" de "instituciones asociadas", la formación de actitudes de respeto institucional en los residentes, los controles periódicos de parte de profesores de la cátedra, la construcción de proyectos articulados con las instituciones asociadas a la práctica y las parejas pedagógicas. La formación en la práctica como problema y responsabilidad compartida implica, según la autora, el desafío de construir un modelo colaborativo entre el centro de formación inicial y la Escuela asociada a la práctica.

Linston y Zeichner (2003) destacan la importancia de formular un contrato consensuado en donde se articulen las acciones, proyectos, prácticas de gestión y organización de la práctica que integren el trabajo colaborativo en ambas instituciones. Diker y Terigi (1997) aportan ideas en la misma línea de razonamiento; la práctica profesional debe ser el centro, el lugar desde donde los objetivos de formación se trabajan constantemente.

A nivel internacional, estudios realizados en América Latina por Sanjurjo (2012), Terigi (2006), Vaillant (2015), Urrutia (2011), en España por Tejada Fernández y Ruiz Bueno (2013) y en Canadá por Correa Molina (2011), advierten sobre la necesidad de colocar en el centro de toda política de formación de profesores la formación práctica y el diseño de redes o mapas de instituciones asociadas a los centros de formación inicial que participan en la etapa de formación. Las evidencias de estos estudios indican que la construcción del conocimiento profesional en los futuros docentes debe comenzar en la etapa de formación inicial y continuar en los espacios de las prácticas.

Uno de los aspectos fundamentales a considerar es la necesidad de generar dispositivos que promuevan la reflexión sistemática, la investigación sobre la práctica y la construcción del rol docente. Tal vez la principal dificultad a superar en Uruguay y otros países de América Latina sea la persistencia del modelo histórico de práctica docente basado en la práctica individual que reproduce hábitos tradicionales y modelos acríticos de la profesión docente. (PÉREZ GÓMEZ, 1996; RODRÍGUEZ ZIDÁN; GRILLI; 2013; GRILLI; SILVA, 2015).

Este modelo histórico de formación individual de los futuros docentes puede ser mejor comprendido a partir del uso de ciertas categorías de análisis que explican cómo la Escuela reproduce prácticas, modelos de hacer y de actuar, regularidades y formas de concebir la construcción del rol docente. Estos rasgos han sido caracterizados como "formatos escolares" (TERIGI, 2006,),

6 Denominación de un espacio de formación donde se analizan las prácticas docentes en la República Argentina. 
"gramáticas escolares" (TYACK; CUBAN, 2001) o lo que Dubet denomina "programas institucionales". (DUBET, 2005).

Sea cual sea la denominación que le demos, las tres categorías señaladas comparten la idea de que los viejos modelos institucionales asociados a los orígenes de los sistemas educativos se perpetuán y reproducen en disonancia con los cambios producidos en las sociedades actuales.

\section{CUADRO 1 - DISPOSITIVOS PARA REFORMAR LA RELACIÓN TEORÍA Y PRÁCTICA}

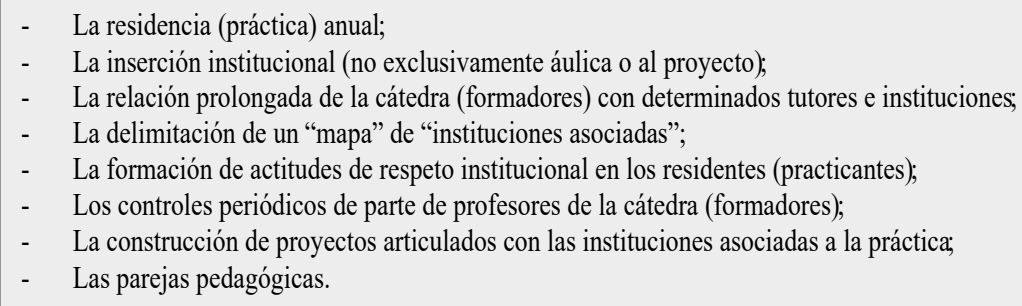

FUENTE: Elaboración propia con base en Sanjurjo (2012).

En base a esto, cambiar el viejo formato escolar de la práctica docente es un desafío que los sistemas de formación deben asumir. En tal sentido, las contribuciones de Sanjurjo (2012, p. 2), en torno a la generación de nuevos dispositivos de formación, son muy valiosas ya que propone específicamente instrumentos para la reforma de las prácticas y la transformación de la relación entre la teoría y la práctica profesional. Algunos de las estrategias a tener en cuenta para consolidar este nuevo modelo de relacionamiento entre la teoría y la práctica se resumen en el siguiente esquema (Cuadro 2).

\section{CUADRO 2 - RASGOS O MARCAS DEL MODELO HISTÓRICO DE FORMACIÓN DE PROFESORES}



- Relación pedagógica entendida como práctica individual;

- Desconexión entre la Escuela de práctica y la institución formadora;

- Divorcio entre la teoría y la enseñanza en contexto real;

- Prácticas pedagógicas fragmentadas en clases breves (45 minutos), reproduciendo un modelo isomórfico con los institutos de formación docente;

- Escaso tiempo en la institución de práctica para la investigación y la reflexión colectiva;

- Ausencia de coordinación entre la dirección de las Escuelas de prácticay el centro de formación inicial;

- Dificultades para que los practicantes de profesorado dispongan en tiempo y forma de profesores adscriptores (orientadores), como tutores de la práctica;

- $\quad$ Asociar lo educativo exclusivamente a la práctica de aula.

FUENTE: Los autores (2016). 
Organizar la vida de la "práctica docente", desde un nuevo formato o programa, implica reconocer la existencias de marcas (TERIGI, 2006) o "elementos permanentes y recurrencias" que hacen a la unidad y a la configuración histórica del viejo formato escolar. (ALLIAUD, 2006). Estos elementos organizan y producen los modos tradicionales de hacer y de actuar en contexto de formación de futuros profesores. En un trabajo de investigación sobre el dispositivo parejas pedagógicas en Uruguay (RODRÍGUEZ ZIDÁN; GRILLI, 2013), se señalan algunas de estas marcas del modelo tradicional de formación de profesores (Cuadro 2).

De acuerdo a lo expuesto, partimos de la hipótesis que una de las claves para lograr un cambio significativo en la cantidad y calidad de profesores que el sistema educativo reclama es construir un nuevo formato de Escuela de práctica basado en la participación del practicante en diferentes círculos y espacios de formación: enseñanza de aula, proyectos didácticas interdisciplinarios, dinamizador de la vida institucional, comprometido en proyectos de extensión, investigador de sus propias prácticas y participante activo de una comunidad de aprendizaje.

Para transformar el modo histórico de formación de profesores y transitar hacia un nuevo formato del núcleo práctica profesional será necesario generar nuevas marcas y huellas en los futuros educadores desde un nuevo diseño institucional. Un camino posible para lograr esta transformación es comenzar por problematizar, situar y contextualizar la formación de los futuros educadores en un nuevo formato de institución de práctica.

La literatura especializada coincide en denominar a este nuevo modelo de articulación como "resonancia colaborativa" entre las instituciones involucradas en contraposición al modelo histórico denominado "disonancia critica", el cual, según varios expertos e investigadores en educación, ha fracasado. (VAILLANT; MARCELO, 2015).

En este nuevo diseño interinstitucional se priorizan el ambiente y la cultura de colaboración, el trabajo por proyectos, la integración de la familia y los agentes comunitarios y el trabajo en coordinación permanente, todo lo cual fortalece los vínculos entre ambas instituciones.

Los centros educativos que trabajan en resonancia compartida producirán, como hipótesis, un efecto de motivación y promoción de buenas prácticas docentes que alentarán procesos innovadores en la formación de profesores. 


\section{¿Cómo lograr resonancia colaborativa entre las instituciones de formación inicial y las instituciones de práctica?}

La experiencia internacional indica que el compromiso escrito y la firma de acuerdos de colaboración mutua es uno de los caminos a implementar. Linston y Zeichner (2003) destacan, para Inglaterra, la importancia de formular un contrato consensuado en donde se articulen las acciones, proyectos, prácticas de gestión y organización de la práctica docente que acuerdan ambas instituciones. Tanto en Suecia, como en el Reino Unido, según un estudio comparado realizado por Vaillant y Manso (2012, p. 7), las instituciones de educación superior que deseen ofrecer programas de formación inicial de docentes deben asociarse con Escuelas para organizar un programa de formación. En el esquema del recuadro siguiente señalamos algunas de las características principales del modelo que estamos analizando (Cuadro 3).

\section{CUADRO 3 - RESONANCIA COLABORATIVA ENTRE INSTITUCIONES EDUCATIVAS}

- Se crean alianzas estables entre los programas y los centros educativos de Práctica;

- Se involucra activamente a los equipos docentes de las escuelas en el diseño, el funcionamiento y la toma de decisiones;

- Se evalúan las prácticas, y se llevan a cabo acciones para enfrentar los desafíos que surgen de la evaluación;

- Las escuelas, y sus docentes, son seleccionadas como colaboradoras con criterios de calidad;

- Se desarrollan estándares que reflejan lo que se debe esperar de las experiencias de práctica criterios de calidad;

- El trabajo conjunto entre las instituciones formadoras y los centros educativos, tanto en la planificación de la formación, como en la selección de los estudiantes y su evaluación se realiza de acuerdo a los estándares;

- Énfasis en la inmersión de los estudiantes en formación en equipos de trabajo coordinados y constituidos por profesores de la universidad y de los centros;

- Seleccionar y capacitar a los supervisores de las practicas, Co-Formadores y Tutores;

- $\quad$ Profesores supervisores de las prácticas efectivos, evaluados y efectivamente recompensados en su desempeño;

- Acuerdo explícitos y permanentes de colaboración entre ambas instituciones.

FUENTE: Elaboración propia en base a Vaillant y Manso (2012), Rodríguez Zidán y Grilli (2013) y Grilli y Silva (2015).

En Argentina se viene desarrollando, a partir de los Lineamientos Curriculares Nacionales para la Formación Docente Inicial, año 2014, una política definida en torno a la necesidad de establecer acuerdos y nexos significativos entre las instituciones formadoras, la Escuela de práctica y otras instituciones 
que podrían participar en red en este nuevo formato de relacionamiento para potenciar la práctica profesional reflexiva. Se señala, en el marco de estas nuevas experiencias: "Las Prácticas y Residencias pedagógicas requieren de un proyecto de trabajo interinstitucional que involucre tanto a las escuelas y organismos sociales como al Instituto Superior". (MECyT, 2014, p. 18).

Esta nueva modalidad implica pensar en redes de colaboración entre docentes, formadores de docentes y estudiantes:

\begin{abstract}
Las redes entre Institutos Superiores y Escuelas asociadas implica la participación activa de los docentes de las escuelas, en un proyecto compartido, que involucra al tramo de la formación en el cual los futuros docentes desarrollan sus primeras experiencias docentes. El ejercicio de este rol requiere, asimismo, que tanto los docentes orientadores de las escuelas como los docentes del Instituto que intervienen en las "Prácticas y Residencia" sean especialmente capacitados para conformar un equipo de trabajo mancomunado en el acompañamiento pedagógico de los estudiantes. (MECyT, 2014, art. 66, p. 35).
\end{abstract}

En España la evidencia recogida en varias investigaciones corrobora que la existencia de centros educativos asignados como Escuela de práctica para la formación inicial de profesores favorece el desarrollo de importantes competencias docentes al promover la conformación de comunidades de aprendizajes y nuevas experiencias de trasformación de la enseñanza. (ANECA, 2009).

En Chile desde el año 2010 las políticas de formación de profesores han decidido impulsar y consolidar " [...] redes de escuelas, liceos y colegios como campos de práctica profesional para los profesores en formación". (MINEDUC, 2010, p. 7; CONTRERAS et. al, 2010). Investigaciones llevadas a cabo con directivos de centros de práctica reportan la importancia que le otorgan al hecho de ser impulsores de liderazgos pedagógicos, señalando la potenciación de las expectativas de asegurar que los estudiantes de las Escuelas de práctica aprendan. (MONTECINOS et al., 2013, p. 23).

\title{
Modelos de formación docente en Uruguay
}

Uruguay se ha caracterizado por tener una carrera de profesorado que adhiere al llamado modelo simultáneo de formación docente. (OCDE, 2009). 
En este modelo la práctica docente adquiere relevancia desde el comienzo de la formación inicial y se da en paralelo a la formación teórica. En otros términos, la formación pedagógica y disciplinar del profesor se da en simultáneo con el ejercicio pre-profesional o práctica docente. De esta manera se ve facilitado la aplicación de la teoría en la práctica la que, a su vez, crea o modifica la teoría. El modelo simultáneo se convierte así en un muy buen soporte para el desarrollo de una relación dialéctica entre teoría y práctica (GRILLI; SILVA, 2015), y con ello también para el desarrollo de lo que algunos autores denominan concepción crítica o emancipatoria de la práctica. (BAQUERO MÁSMELA, 2006). Bajo esta concepción se apunta a desarrollar una práctica docente que, analizando de manera crítica lo que sucede en la Escuela de práctica, genere cambios en los ciudadanos para mejorar la sociedad.

Vale la pena volver aquí a insistir en la importancia de promover una práctica docente innovadora identificada con el modelo de resonancia colaborativa. (ESCOBAR, 2007). En este modelo la Escuela de práctica y el centro de formación inicial de docentes deben convertirse en lugares donde se indague, de forma sistemática y crítica, sobre los procesos de enseñanza, los resultados de aprendizaje y la relación que estos tienen con el funcionamiento y organización del centro educativo.

El éxito de un modelo simultáneo que apunta a una relación dialéctica entre teoría y práctica, buscando desarrollar una "crítica emancipadora de la práctica", tiene como soporte o base la relación que se consigue configurar entre las dos instituciones educativas participantes de la formación docente.

Como dijimos al inicio, en Uruguay la formación pública de docentes está reglamentada por el Sistema Único Nacional de Formación Docente (SUNFD) (Plan 2008). Bajo la normativa de este Plan de estudios cualquier centro educativo de nivel medio puede ser receptor de estudiantes practicantes de profesorado. La única experiencia en sentido contrario a esto se dio en los Ce.R.Ps ${ }^{7}$ durante los Planes de estudio 1997 y 2005 que asignaban determinados centros de educación media como Escuela de práctica para los estudiantes que cursaban el $4^{\circ}$ año de su carrera.

A pesar del cambio de plan que se dio con el SUNFD, en la actualidad el Ce.R.P del Litoral (delimitación institucional y geográfico del estudio que estamos presentando) mantiene concentrados en un único centro educativo medio a más del $80 \%$ de los practicantes de $4^{\circ}$ año de las 15 carreras de profesorado que contiene la oferta curricular.

7 Ce.R.Ps: Centros Regionales de Profesores, son 6 institutos, creados a partir de 1997, atienden la formación de profesores en todo el país, menos Montevideo. (VAILLANT; WETTSTEIN, 2007). 
Una figura fundamental en las instancias de práctica docente es la del supervisor. En el SUNFD el supervisor de la práctica es el profesor de las asignaturas Didáctica: Didáctica I en $2^{\circ}$, Didáctica II en $3^{\circ}$ y Didáctica III en $4^{\circ}$ año. En los dos primeros años se desarrolla una práctica co-supervisada: además del profesor de Didáctica existe el rol del profesor adscriptor. Este docente es el titular del grupo de nivel educativo medio donde el estudiante de formación docente hace su práctica. La supervisión entonces en estos dos años se realiza bajo la supervisión de dos profesores: por un lado el profesor de didáctica y por otro el profesor adscriptor asignado. Con ellos, el practicante comparte las tareas docentes: programación de cursos, selección de estrategias, evaluación de aprendizaje, dictado de clase, entre otras. Por otra parte, en un nivel superior de la formación inicial (en la práctica de 4to. año) el estudiante de profesorado asume un grupo de nivel educativo medio, desarrollando o cumpliendo en él, de forma autónoma, todas las funciones que le compete a un docente. Por estas características, las experiencias, marcas o huellas, que esta práctica deja en el estudiante, son de gran significación e impacto, y es a esta práctica que nos referiremos particularmente en el trabajo que realizamos.

\section{Diseño metodológico}

La investigación acumulada sobre métodos y validez en las ciencias sociales muestra un consenso cada vez más generalizado sobre la necesidad de maximizar la objetivación (reduciendo las fuentes de invalidez) de procesos sociales complejos como lo son el análisis de las prácticas pedagógicas y los modelos de formación de profesores. El enfoque aplicado en este estudio se basa en la articulación de métodos cuantitativos y cualitativos del tipo "multimetho dapproach" (KELLE, 2001), o aproximaciones multimétodo que implementan diseños basados en el bricoleur metodológico. (DENZIN; LINCOLN, 2012; CRESWELL; PLANO, 2011). Este procedimiento es apropiado para investigar las prácticas educativas, los procesos de cambio y el desarrollo de modelos de formación inicial de docentes.

Para esta investigación el universo de estudio está ubicado geográficamente en el departamento de Salto Uruguay: Centro Regional de Profesores del Litoral, segundo centro de formación de profesores más importante del país en términos de matrícula y oferta curricular. En función de la perspectiva metodológica señalada, se optó por la aplicación de un diseño con modelo mixto de investigación social al incluir instrumentos del paradigma positivista y naturalista en 
el proceso de recolección de datos. El diseño fue de tipo concurrente CUAL + CUAN (CAMERON, 2009) es decir, aplicándose simultáneamente los instrumentos seleccionados. La muestra de sujetos (de carácter no probabilística) se integró por 122 estudiantes practicantes de profesorado y 75 profesores de centros de práctica.

Para el abordaje cuantitativo se diseñó un cuestionario con variables de opinión, preguntas cerradas, abiertas y escalas tipo Lickert. El enfoque cualitativo se implementó mediante una muestra por juicio o teórica (MAXWELL, 1996) de informantes calificados (egresados de distintos planes de estudio; directores; profesores de didáctica y personal administrativo). El estudio, a partir de estos aportes, contrastó y validó la información recogida de 13 entrevistas en profundidad con el análisis de las opiniones de diferentes grupos de docentes y estudiantes encuestados (Cuadro 4). Una selección de las preguntas que integraron la guía de entrevista se presenta en el Anexo.

CUADRO 4 - PERFIL DE LOS ENTREVISTADOS

\begin{tabular}{|c|c|c|}
\hline Rol & Plan de Formación Docente & Especialidad \\
\hline Docente Secundaria & 1997 & Biología \\
\hline Docente Secundaria & 1997 & Historia \\
\hline Docente Secundaria & 2008 & Biología \\
\hline Docente Secundaria & 1997 & Química \\
\hline Docente Secundaria & 2005 & Inglés \\
\hline Supervisor/Profesor de didáctica & $1997 / 2005 / 2008$ & Geografía \\
\hline Supervisor/Profesor de didáctica & $1997 / 2005 / 2008$ & Inglés \\
\hline Supervisor/Profesor de didáctica & $1997 / 2005 / 2008$ & Idioma Español \\
\hline Supervisor/Profesor de didáctica & $2005 / 2008$ & Historia \\
\hline $\begin{array}{l}\text { Director/Centro de Formación } \\
\text { Inicial }\end{array}$ & $1997 / 2005 / 2008$ & Geografía \\
\hline Director Escuela de práctica & $1997 / 2005 / 2008$ & Historia \\
\hline Ayudante Laboratorio & 2008 & Química \\
\hline Personal administrativo & $1997 / 2005 / 2008$ & -------- \\
\hline
\end{tabular}

FUENTE: Los autores (2016).

El principal procedimiento de validez, considerando las recomendaciones de Denzin y Lincoln (2012), fue la triangulación de datos (cruzando relatos entre diferentes entrevistados) y triangulación metodológica entre métodos, contrastando datos estadísticos con el discurso de los docentes. 


\section{Resultados}

Entre los principales resultados del estudio debe destacarse como hallazgo una opinión positiva de la modalidad en un único centro. Los datos relevados indican que los grupos más favorables a esta modalidad son los alumnos de $3 \mathrm{er}$. año (67\%, N, 50 casos), estudiantes de 4to año (64\%, N, 72 casos), profesores no adscriptores $(69 \%, \mathrm{~N}, 26$ casos) y profesores adscriptores (57\%, N, 49 casos).

Los datos del Gráfico 1 muestran que los grupos más favorables a esta modalidad son los alumnos de 3 er. año y los profesores de educación media encuestados. También puede apreciarse que las opiniones están más divididas entre los profesores orientadores de la práctica incluidos adscriptores de la población seleccionada.

\section{GRÁFICO 1 - CON RELACIÓN A QUE LOS ESTUDIANTES CURSEN LA PRÁCTICA CONCENTRADOS EN UNA ESCUELA DE PRÁCTICA: ¿CONSIDERA QUE ES UNA MODALIDAD QUE DEBERÍA SER DESARROLLADA EN EL FUTURO DE LA FORMACIÓN DOCENTE?}

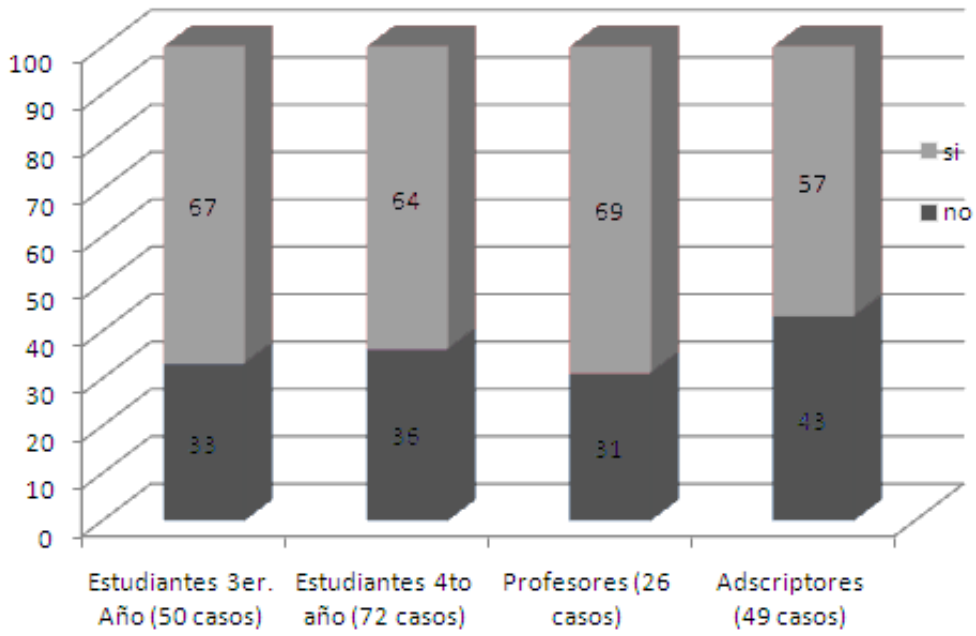

FUENTE: Los autores (2016).

La mayoría relativa de los encuestados (66\%) sostienen que la práctica docente debería realizarse en una sola institución.

A efectos de profundizar un poco más en el análisis se realizó una prueba chi cuadrado para observar si las diferencias entre los grupos - con respecto a 
la opinión sobre el hecho de considerar que la EP debería realizarse en un solo centro - era significativa.

Los datos que comprueban la existencia de diferencias significativas entre estudiantes 3er. y de 4 to año de prácticas $\chi^{2}(1, N=122)=3.68, p<.05$.), entre los docentes de educación media según su condición de supervisores o no de los practicantes $\chi^{2}(1, N=75)=1,05 p<.05$.) y entre el grupo de estudiantes practicantes y el colectivo de docentes encuestado $\left.\chi^{2}(1, N=197)=1,76 p<.05\right)$.

El grupo de docentes con mayores observaciones y discrepancias con respecto a la modalidad en un sólo centro de Escuela de práctica fue el colectivo de docentes co-supervisores: los adscriptores.

\section{Valoraciones sobre la práctica docente}

Posteriormente al análisis estadísticos de las opiniones, nuestro interés fue profundizar sobre la experiencia observada, recuperando la voz y las valoraciones de los estudiantes, docentes e informantes calificados entrevistados. Se construyeron cinco dimensiones consideradas ejes principales del análisis cualitativo, tal como lo representa la Figura 1.

\section{FIGURA 1 - DIMENSIONES DE ANÁLISIS DE CENTRO EDUCATIVO DE PRÁCTICA}

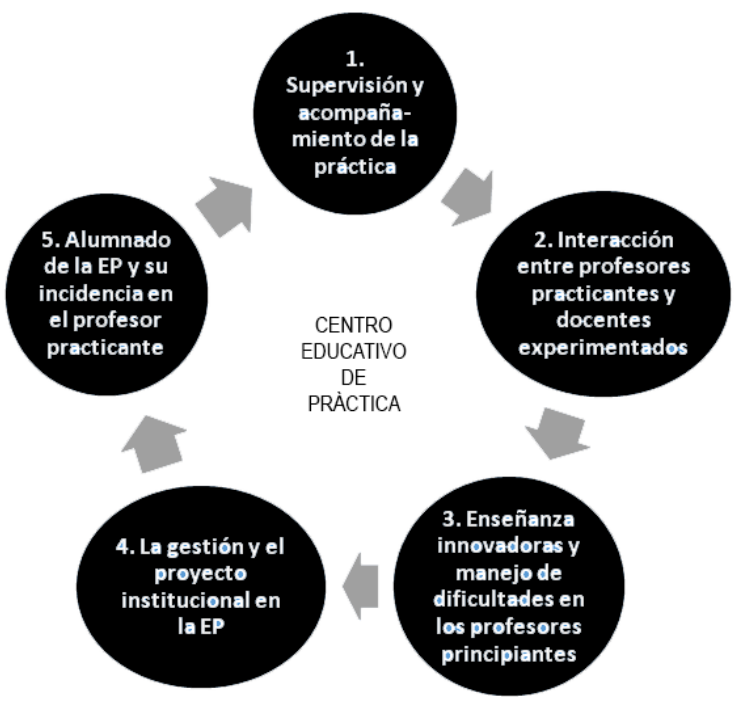

FUENTE: Los autores (2016). 
Desarrollamos a continuación el análisis de cada una de estas dimensiones, destacando como contribuyen o, en su defecto, obstaculizan el desarrollo de la experiencia de formación en una única Escuela de práctica.

\section{Supervisión y acompañamiento de la práctica}

Como sostiene Escobar (2007), la práctica profesional del docente se caracteriza por la complejidad, singularidad y simultaneidad de las interacciones que en ella se dan, las que se desarrollan en el marco de diversas concepciones, dilemas y obstáculos. En este sentido la tarea que cumple el profesor supervisor de la práctica (el profesor de Didáctica en nuestro sistema de formación docente), es fundamental. Correa Molina (2011) señala que el supervisor, por poseer una vasta experiencia docente, conoce la realidad del medio escolar y al mismo tiempo por representar a la institución formadora conoce los objetivos y los contenidos de la didáctica que ahí se enseñan.

Es el profesor de didáctica uno de los principales responsables de generar condiciones de funcionamiento de la práctica para que se vaya dando en el transcurso de la misma los necesarios procesos de análisis, discusión y clarificación de los supuestos e ideas educativas. Se busca así que las mismas no interfieran en las posibilidades reales de aprendizaje en terreno. (WIDEEN; MAYER-SMITH; MOON, 1998; ZANTING; VERLOOP; VERMUNT, 2001; ZEICHNER, 1996). Haciendo esto, el supervisor de práctica desarrolla lo que podemos denominar una función de acompañamiento: ayuda los practicantes a clarificar las teorías personales que inciden en el desempeño profesional, promoviendo la adopción de una postura reflexiva e investigativa de las prácticas de aula.

La teoría pedagógica necesariamente debe articularse a las situaciones educativas que las requieran, para fundamentar la práctica. Como sostiene Lessard y Bourdoncle (1998), una formación profesional no puede apoyarse sólo en saberes fundamentados por la práctica, se requieren conceptos y teoría para confrontar con la experiencia. Para que todo esto se dé en buena forma la función de acompañamiento que desempeña el profesor supervisor es fundamental.

Además de acompañar, el supervisor - y valga la redundancia - cumple una segunda función: supervisa. Es necesario que en determinados momentos del proceso se tome distancia para evaluar el grado de desarrollo adquirido de las competencias que se buscaron generar en el acompañamiento realizado. Con esto se tiene entonces por objetivo constatar aprendizajes: valorar el grado de desarrollo adquirido en las competencias docentes trazadas como meta.

Como podemos ver en las opiniones recogidas para la dimensión 1, diferentes actores institucionales (directores, estudiantes, personal de laboratorio) valoran positivamente la concentración de estudiantes de práctica de un mismo 
nivel de la carrera, en un único centro (Cuadro 5). Evidentemente esto facilita el contacto y seguimiento del supervisor con sus profesores practicantes, al tiempo que facilita las necesarias coordinaciones entre las dos Instituciones educativas involucradas con la formación práctica de los futuros docentes.

No obstante algunos estudiantes señalaron como aspecto negativo la desvalorización de los profesores practicantes por parte de algunos actores consultados. Posiblemente una causa del hecho constatado sea lo que Kaddouri y Vandroz (2008) señala como falta de coherencia entre el proyecto identitario que la universidad o instituto de formación inicial y la Escuela de práctica.

CUADRO 5 - SUPERVISIÓN Y ACOMPAÑAMIENTO DE LA PRÁCTICA POR PARTE DEL CENTRO FORMADOR Y LA ESCUELA DE PRÁCTICA

\begin{tabular}{|c|c|}
\hline Aspectos que contribuyen a fortalecer el modelo & \begin{tabular}{|c|} 
Aspectos que dificultan la \\
implementación del modelo
\end{tabular} \\
\hline $\begin{array}{l}\text { "Estando en un solo centro se facilita el conocimiento del } \\
\text { funcionamiento del mismo. En cambio estando en los liceos } \\
\text { cada uno tiene sus características distintas y nosotros no } \\
\text { estamos en contacto tan asiduo con la dirección, como } \\
\text { se estaba antes en los otros planes. Con los adscriptos } \\
\text { incluso... que levantaban el teléfono y nos llamaban por } \\
\text { alguna situación con algún practicante... se pierden esas } \\
\text { cosas [...]." (Profesora de Didáctica, especialidad Historia) } \\
\text { "Por estar en la órbita del Consejo Técnico Profesional } \\
\text { tiene alumnos de todo tipo; aquí se puede visualizar } \\
\text { fácilmente la problemática de otros lados: aprendizaje, } \\
\text { desvinculación, violencia, etc. como en todas las } \\
\text { instituciones educativas pero acá hay más gente dispuesta } \\
\text { para solucionar los problemas y estamos amalgamados a } \\
\text { un Centro de Formación Docente, somos centros hermanos } \\
\text { que nacimos el mismo día y con equipos de dirección que } \\
\text { tienen el mismo planteo y forma de pensar a los efectos de } \\
\text { la práctica." (Director de Escuela de práctica) } \\
\text { "Yo hice mi práctica aislada en un liceo, sin otros } \\
\text { compañeros que tuvieran realizando tareas similares a las } \\
\text { mías... no podia compartir. La práctica es más organizada, } \\
\text { controlada, fiscalizada, tenemos los mismos recursos, } \\
\text { podemos comparar... es mejor todos en un mismo Centro } \\
\text { Educativo." (Ayudante preparador de laboratorio del } \\
\text { Escuela de práctica) }\end{array}$ & $\begin{array}{l}\text { "[...] sentiamos que adscrip- } \\
\text { tos y directores del CP nos } \\
\text { trataban de tal forma que te } \\
\text { hacian notar tu posición de } \\
\text { estudiante. [...] En algunos } \\
\text { casos te pedian que realiza- } \\
\text { ras tareas que eran exclusi- } \\
\text { vamente de la adscripción, } \\
\text { como desvalorizándote, } \\
\text { trasladándote a un puesto } \\
\text { de empleada o ayudante de } \\
\text { adscripta. [...] Creo que se } \\
\text { acostumbran demasiado a } \\
\text { que todos los años reciben } \\
\text { practicantes, que en vez de } \\
\text { verlos como estudiantes en } \\
\text { plena formación a los que de- } \\
\text { ben tratar de ayudar y volcar } \\
\text { su experiencia, nos visualizan } \\
\text { como incompetentes a los que } \\
\text { deben exigir." (Egresada es- } \\
\text { pecialidad Biología) }\end{array}$ \\
\hline
\end{tabular}

FUENTE: Los autores (2016). 
Interacción entre profesores practicantes y docentes experimentados

Como señala Correa Molina (2011), los profesores con experiencia no pueden seguir siendo considerados como agentes silenciosos de la formación docente, o como simples prestadores de servicio que facilitan el acceso a sus clases. La contribución que ellos pueden hacer a la formación de los futuros colegas es relevante y por tanto no debería dejarse de lado o menospreciarse.

La concentración de estudiantes practicantes en una Escuela de práctica implicó que en el turno matutino de la misma casi todas las asignaturas de los grupos de práctica estuviesen a cargo de profesores practicantes. Como consecuencia, las instancias de intercambio pedagógico y de coordinación de acciones, se implementa de forma permanente entre docentes en formación. Esto es señalado como una debilidad por parte de algunos de los entrevistados como podemos ver en una docente egresada de Historia (Cuadro 5).

Paradójicamente y al mismo tiempo, la concentración de estudiantes practicantes en una única Escuela de práctica y en un turno especifico, trajo aparejado una mayor y mejor coordinación entre los profesores de los grupos. Como vemos en las valoraciones positivas que recogemos los practicantes señalan que, al interactuar con otros profesores que también son practicantes, desarrollan una experiencia en un plano de igualdad que los lleva a sentirse más seguros y cómodos para plantear con fluidez las dificultades y problemas que hay que resolver.

Enseñanza innovadora y atención de dificultades propias de los profesores principiantes

Los datos recogidos permiten visualizar que el proceso de conformación de comunidades de aprendizaje donde se desarrolla una "cultura de colaboración" (IMBERNÓN, 1994; MARCELO, 2000), se ve favorecido por la concentración de estudiantes practicantes en una sola escuela (evitando la dispersión geográfica y curricular) donde es posible implementar otros dispositivos de formación como la pareja pedagógica y las visitas y devoluciones colectivas de clases. (RODRÍGUEZ ZIDÁN; GRILLI, 2013; GRILLI; SILVA, 2015). Se observa una buena motivación docente, componente básico de la satisfacción personal y de la mejora educativa. (HERRÁN, 2008). Los testimonios señalados por diferentes actores consultados (egresados, personal administrativo, ayudante preparador de laboratorio), coinciden en destacar el valor de los profesores practicantes por ser innovadores: prueban y ensayan estrategias y recursos, en la búsqueda de nuevas respuestas a los problemas educativos que se presentan. También se menciona el buen conocimiento que suelen tener los profesores practicantes de 
CUADRO 6 - INTERACCIÓN ENTRE PRACTICANTES Y DOCENTES EXPERIMENTADOS

\begin{tabular}{|c|c|}
\hline Aspectos que contribuyen a fortalecer el modelo & \begin{tabular}{|c|}
$\begin{array}{c}\text { Aspectos que dificultan } \\
\text { la implementación del } \\
\text { modelo }\end{array}$ \\
\end{tabular} \\
\hline $\begin{array}{l}\text { "[...] en la Escuela de práctica el practicante se siente más } \\
\text { seguro, estando con sus compañeros que a su vez son colegas. } \\
\text { En cambio en los liceos, el practicante por lo general se } \\
\text { queda afuera, no entra por ejemplo a la Sala de Profesores. } \\
\text { No entra, tiene temor, tiene vergüenza... solamente los que } \\
\text { tienen más capacidad para relacionarse son los que ingresan } \\
\text { a la Sala de Profesores. En cambio en la Escuela de práctica, } \\
\text { se sienten más profesores' diríamos." (Profesora de Didáctica, } \\
\text { especialidad Historia) } \\
\text { "[...] te sentís más acompañado y sustentado anímicamente, } \\
\text { por el hecho de no llegar a un lugar donde todo el mundo te } \\
\text { es extraño, especialmente en este momento de la carrera donde } \\
\text { el estrés, los nervios y la ansiedad actúan como organismos } \\
\text { oportunistas." (Egresada, especialidad Biología) } \\
\text { "Se hace más fluido la comunicación y el intercambio cuando } \\
\text { hay practicantes; ellos generalmente conocen más y mejor } \\
\text { sobre la situación de los alumnos del grupo que nosotros los } \\
\text { profesores efectivos y todo se comparte en las coordinaciones." } \\
\text { (Egresado, especialidad Biología) }\end{array}$ & $\begin{array}{l}\text { "[...] estar en un solo } \\
\text { turno hacía que la mayo- } \\
\text { ría de los colegas fueran } \\
\text { también practicantes y } \\
\text { no docentes egresados y } \\
\text { de larga trayectoria con } \\
\text { los cuales intercambiar } \\
\text { opiniones o enseñarnos } \\
\text { cosas del día a día, de } \\
\text { la práctica." (Egresada, } \\
\text { especialidad Historia) }\end{array}$ \\
\hline
\end{tabular}

FUENTE: Los autores (2016).

sus estudiantes: las condiciones y problemas generales y circunstanciales por los que puedan estar pasando (Cuadro 7).

Por otra parte está muy presente, en esta tercera dimensión analizada, dos de las dificultades señaladas en los profesores principiantes: la disciplina en el aula y los problemas con determinados alumnos. (VEENMAN, 1984, 1988). Estas dificultades se manifiestan claramente en la formación inicial cuando los practicantes de $4^{\circ}$ año asumen un grupo como responsables. La conformación de comunidades educativas en el turno donde funciona la práctica docente ha contribuido positivamente a encontrar soluciones a estas dificultades, comunes en el docente inexperiente. No obstante es indudable que el hecho de que la mayoría de los profesores de los grupos del turno matutino, son practicantes, las dificultades de control de la disciplina en el aula y el manejo de situaciones con determinados alumnos se hacen más notorios. Este tipo de valoración negativa de la práctica de enseñanza con los estudiantes que analizamos en la dimensión 3 (Cuadro 7), es mencionada por varios de los informantes entrevistados. 
CUADRO 7 - PRÁCTICAS DE ENSEÑANZA CON LOS ESTUDIANTES DEL NIVEL EDUCATIVO MEDIO

\begin{tabular}{|c|c|}
\hline Aspectos que contribuyen a fortalecer el modelo & $\begin{array}{c}\text { Aspectos que dificultan } \\
\text { la implementación del } \\
\text { modelo }\end{array}$ \\
\hline $\begin{array}{l}\text { "Los problemas en una Escuela de práctica no pasan } \\
\text { desapercibidos, los practicantes hacen un control mayor, se } \\
\text { quedan más tiempo en la institución y conocen mejor a sus } \\
\text { estudiantes." (Egresado, especialidad Biología) } \\
\text { "Lo que observo con los practicantes es que siempre están } \\
\text { viendo actividades nuevas, prácticas nuevas, usan recursos } \\
\text { que sí comparamos con docentes que hace años que están en } \\
\text { la institución no los usan. Vemos que se ha visto enriquecido } \\
\text { el Centro porque es variada la actividad, la propuesta. Los } \\
\text { muchachos más jóvenes no le tienen miedos a los recursos." } \\
\text { (Ayudante preparador de laboratorio de la Escuela de práctica) } \\
\text { "Cuando hacemos las inscripciones en diciembre, los tres } \\
\text { primeros dias ya se nos agota el turno matutino. Te cuento } \\
\text { una anécdota: los padres me decían que hermoso es ver tener } \\
\text { profesores jóvenes y yo les decía que ellos tenían que valorar } \\
\text { pues están en la misma generación y por esto los pueden } \\
\text { entender mejor; era una reunión con un grupo numerosísimo } \\
\text { de padres y ellos estaban muy contentos de tener profesores } \\
\text { practicantes. También el ausentismo docente que como dijimos } \\
\text { es muy bajo en la mañana actúa para que los padres prefieran } \\
\text { que sus hijos estén en este turno." (Personal administrativo de } \\
\text { la Escuela de práctica) }\end{array}$ & $\begin{array}{l}\text { “A veces pasaba que al } \\
\text { ser tantos practicantes se } \\
\text { escuchaban inconvenien- } \\
\text { tes con los profesores que } \\
\text { veían carencias concep- } \\
\text { tuales o de manejo en los } \\
\text { practicantes." (Egresada, } \\
\text { especialidad Inglés) } \\
\text { "No manejan, no tienen } \\
\text { dominio de grupo yene- } \\
\text { ralmente en primer año } \\
\text { se les distorsionan; los } \\
\text { alumnos son muy niños y } \\
\text { por eso hay que tomar con } \\
\text { más firmeza las situacio- } \\
\text { nes." (Personal adminis- } \\
\text { trativo de la Escuela de } \\
\text { práctica) }\end{array}$ \\
\hline
\end{tabular}

FUENTE: Los autores (2016).

La gestión y proyecto institucional en la Escuela de práctica

Un nuevo aspecto a evaluar es el siguiente: ¿en qué medida el tamaño de la Escuela de práctica - en función del número de estudiantes practicantes y de orientadores, de los turnos de la escuela afectados y de la implementación del proyecto institucional de centro por los estudiantes de pedagogía - podría significar un problema para la gestión eficaz del proceso de formación? Para analizar esta pregunta - identificada con la dimensión 4 -, seleccionamos varios testimonios de directores, personal administrativo que gestiona la Escuela de práctica y profesores de educación media que se formaron bajo esta modalidad (Cuadro 8). 
CUADRO 8 - GESTIÓN DE LA ESCUELA DE PRÁCTICA

\begin{tabular}{|c|c|}
\hline $\begin{array}{c}\text { Aspectos que contribuyen a fortalecer el } \\
\text { modelo }\end{array}$ & $\begin{array}{l}\text { Aspectos que dificultan la } \\
\text { implementación del modelo }\end{array}$ \\
\hline $\begin{array}{l}\text { "Yo me pregunto si un secundario que } \\
\text { tiene } 2000 \text { o } 3000 \text { estudiantes y cientos de } \\
\text { profesores con diferentes culturas, diferente } \\
\text { formación, diferentes generaciones, diferentes } \\
\text { compromisos, ¿no es dificil de gestionar? } \\
\text { Yo no creo que esto sea menos dificultoso } \\
\text { que una Escuela de práctica en donde } \\
\text { nuestros estudiantes, practicantes, asistian } \\
\text { a las reuniones de coordinación y estaban } \\
\text { empapados y colaboraban con el Proyecto } \\
\text { de Centro." (Director de Centro Formador de } \\
\text { Profesor) } \\
\text { "Yo creo que si va haber una dificultad } \\
\text { donde hay tantas personas para gestionar la } \\
\text { Escuela, pero no es imposible... con buenas } \\
\text { coordinaciones y buen relacionamiento entre } \\
\text { las dos instituciones, la Escuela de práctica y } \\
\text { el Centro Formador de Docentes, se logra." } \\
\text { (Personal administrativo de la Escuela de } \\
\text { práctica) }\end{array}$ & $\begin{array}{l}\text { "Habia mucho autoritarismo, acatar nor- } \\
\text { mas pre-establecidas, hacerse funcional } \\
\text { a un sistema que ya estaba en funciona- } \\
\text { miento lo que dificulta hacerse autóno- } \\
\text { mo." (Egresada, especialidad Historia) } \\
\text { "[...] si se trata de trabajar con los } 100 \\
\text { practicantes juntos como se ha venido } \\
\text { haciendo, va a ser complicado gestionar } \\
\text { la Escuela; pero si se organiza para tra- } \\
\text { bajar con grupos más pequeños, va a ser } \\
\text { más efectivo. Es cierto que se tendrán que } \\
\text { repetir cosas en cada uno de los grupos } \\
\text { de trabajo, pero se coordinará mejor. [...] } \\
\text { Depende de la gestión, no es algo impo- } \\
\text { sible de realizar." (Egresado, especialidad } \\
\text { Biología) }\end{array}$ \\
\hline
\end{tabular}

FUENTE: Los autores (2016).

La experiencia realizada en Uruguay, en ocasiones, llegó a concentrar hasta cien estudiantes practicantes en un mismo turno. La constatación de este hecho y las evidencias recogidas confirman que esta particular situación representó un gran desafío de gestión y coordinación administrativa para lograr resonancia colaborativa eficiente entre las instituciones. En los límites, la búsqueda de la máxima eficiencia para cumplir con el propósito de formación podría caer en la imposición del modelo de gestión de la Escuela de práctica (en algunos casos identificado por los entrevistados como "autoritario") e inhibir la autonomía docente y la independencia necesaria para la toma de decisiones que hacen a la formación práctica y crítica del futuro profesor de secundaria. En el extremo opuesto, un espacio de formación excesivamente no regulado de la formación dejaría al practicante cautivo de experiencias basadas en su propia rutina individual e itinerante en función de las prácticas de gestión y organización curricular disponibles en el sistema educativo. El caso analizado parecería indicar que una buena práctica de articulación y gestión, entre las dos instituciones, podría ser el camino para la mejora. Una segunda lección del análisis de esta dimensión es la 
consideración del tamaño de la Escuela de práctica: una alternativa a explorar es el diseño de proyectos de resonancia colaborativa en escuelas de práctica de pequeña y mediana escala.

\section{Alumnado de la Escuela de práctica y su incidencia en el profesor practicante}

Una de las críticas u objeciones que se han formulado a la existencia de una Escuela de práctica definida como tal por las propias autoridades del sistema educativo refiere a las características especiales y/o artificiales que la misma adquiere para recibir al profesor practicante. $\mathrm{Al}$ respecto una de las cuestiones mencionadas tiene que ver con el riesgo de seleccionar escuelas con características homogéneas del alumnado desde el punto de vista sociocultural, lo cual inhabilitaría la posibilidad de enfrentar a los practicantes a los desafíos de la enseñanza en contextos sociales heterogéneos y diversos.

Las respuestas de los entrevistados señalan dos aspectos sobre el alumnado de la Escuela de práctica. Por un lado se alude a la diversidad de alumnos existentes en el centro educativo dado que el mismo no está limitado a un radio de la ciudad. En directa vinculación con el punto anterior se menciona la dificultad educativa que enfrenta el practicante ya que varios alumnos del centro de educación media provienen de contextos sociales y culturales carenciados. No obstante, no se ve el hecho como una debilidad en sí misma, por el contrario se lo menciona como una oportunidad para adquirir competencias docentes y como una condición que no es muy diferente a la que existe en otras instituciones educativas de nivel medio (véase Cuadro 9). Por otro lado uno de los egresados plantea como debilidad de la Escuela de práctica la artificialidad que la misma representa: por tener un mejor equipamiento didáctico y grupos de clase con baja cantidad de alumnos, contrasta con la realidad existente en otras instituciones donde luego se trabajará.

El segundo aspecto señalado por algunos de los docentes indagados refiere al tipo de relación profesor-alumno, que se configura en la práctica docente. Se sostiene que el alumnado de la Escuela de práctica a sabiendas de que sus profesores son alumnos de otra institución, establecen una relación con ciertos ribetes de perversidad. En ocasiones, esta realidad podría implicar el surgimiento de vínculos erosionados entre profesor alumno caracterizado por el chantaje o juego de convivencia (ya que los alumnos de secundaria, sabiendo que sus docentes son practicantes y deben rendir una prueba final en clase, podrían utilizar este hecho como un mecanismo de presión). Este hecho habría que confirmarlo con mayores evidencias y observar con mayor atención el grado de incidencia en los resultados y los procesos de enseñanza y aprendizaje. 
CUADRO 9 - ALUMNADO DE LA ESCUELA DE PRÁCTICA Y SU INCIDENCIA EN EL PROFESOR PRACTICANTE

\begin{tabular}{|c|c|}
\hline 1 modelo & $\begin{array}{l}\text { an la } \\
\text { odelo }\end{array}$ \\
\hline $\begin{array}{l}\text { "Acá tenemos alumnos de la campaña y de todo Salto, } \\
\text { como es el único centro no tenemos radio como en } \\
\text { los liceos y por eso tenemos una variedad enorme } \\
\text { de alumnos lo que hace que el practicante se foguee } \\
\text { mucho más." (Personal administrativo de la Escuela } \\
\text { de práctica) } \\
\text { "Hoy las realidades son similares. Yo que trabajo en } \\
\text { distintos Centros Educativos si bien puedo encontrar } \\
\text { alguna diferencia, por ejemplo un trabajo más } \\
\text { tranquilo, alumnos que aportan más, la realidad } \\
\text { hoy por hoy es la misma. Para mi está bien que } \\
\text { comiencen acá con alumnos con carencias, de primer } \\
\text { ciclo, alumnos que hay que organizarlos para poder } \\
\text { estudiar." (Personal administrativo) } \\
\text { "Yo, como recién egresada, trabajo en tres } \\
\text { instituciones, y se cómo manejarme en cada una de } \\
\text { ellas, pues las tres están fundadas en distintos ideales. } \\
\text { Sin embargo, en mi último año conté con un único } \\
\text { centro de práctica, lo cual no representó obstáculo } \\
\text { alguno para desenvolverme en mi trabajo actual." } \\
\text { (Egresada, especialidad Biología) }\end{array}$ & $\begin{array}{l}\text { "Se nos mostró una realidad muy } \\
\text { diferente, hasta desde el punto } \\
\text { de vista edilicio una realidad } \\
\text { confortable, agradable y después } \\
\text { saliamos a otras instituciones } \\
\text { con múltiples carencias, sin } \\
\text { recursos tecnologicos, con grupos } \\
\text { super-numerosos." (Egresada, } \\
\text { especialidad Historia) } \\
\text { "[...] los profesores practicantes } \\
\text { dependen de ellos para salvar el } \\
\text { examen. Esto es una debilidad } \\
\text { hoy en dia... los alumnos juegan' } \\
\text { hacen una especie de extorsión y } \\
\text { los practicantes se sienten como } \\
\text { presionado; la actitud de los } \\
\text { alumnos de UTU, sobre todo los } \\
\text { de } 3 \text { o, cambia en función de si } \\
\text { son practicantes o docentes ya } \\
\text { de años." (Egresado, especialidad } \\
\text { Biología) }\end{array}$ \\
\hline
\end{tabular}

FUENTE: Los autores (2016).

\section{Conclusiones}

A partir del examen de los datos y testimonios recogidos es posible observar que la mayoría de los entrevistados comparten una valoración general positiva del modelo Escuela de práctica: concentración de estudiantes para la realización de la práctica pre-profesional, en un centro educativo. El acuerdo parecería ir más allá del grupo o estamento entrevistado: los profesores de didáctica, los directores de los dos centros educativos, personal administrativo ayudantes de laboratorio y estudiantes de profesorado. No obstante, su implementación demanda importantes desafíos.

Las entrevistas y preguntas abiertas realizadas mediante cuestionario presentan fuertes coincidencias en torno a un concepto que podemos sintetizar 
en la siguiente frase: realizar la práctica en un centro y en el mismo turno, logra conjugar profesores practicantes y profesores profesionales, facilitándose las tareas de acompañamiento y supervisión de los profesores en formación por una mayor resonancia colaborativa entre las instituciones educativas participantes.

Los datos relevados apoyan la idea y la necesidad de generar una mayor y más potente articulación entre el centro educativo de práctica y la institución formadora. Lograr cambios significativos en las prácticas de enseñanza orientadas a la reflexión crítica (TALLAFERRO, 2006), encuentra en la concentración de los profesores practicante en uno o dos centros de práctica, un importante soporte. El estudio muestra la relevancia de lograr una mayor participación de los docentes en comunidades de aprendizaje, por ejemplo aprovechando mejor el espacio de coordinación institucional. La mayoría de los informantes entrevistados destacan que el desarrollo de hábitos y procedimientos de observación y discusión de prácticas educativas, con base en teoría pedagógica que se contextualiza a la realidad del centro, es sustancial para la formación docente.

\section{Discusión}

Los datos analizados apoyan la hipótesis de que la concentración de estudiantes de formación docente en unos pocos centros educativos que sirvan como Escuelas de práctica, facilitan las tareas de supervisión y acompañamiento del profesor en formación, y con ello se mejoraría:

1 - El análisis y la reflexión crítica de la práctica de aula;

2 - La participación de los profesores practicantes en las comunidades de aprendizaje;

3 - La relación dialógica entre teoría pedagógica y práctica docente;

4 - El fortalecimiento de procesos educativos innovadores.

La práctica en una única institución (o eventualmente en dos, dependiendo de la escala y matricula del instituto de formación inicial) facilita el trabajo mancomunado entre los dos establecimientos involucrados en la formación docente. El diálogo y la coordinación entre las instituciones mediante acuerdos y contratos de trabajo y acciones que garanticen un ambiente favorable, se desarrollaría con mayores posibilidades de éxito en la modalidad con centro de práctica que con práctica dispersa en múltiples centros de educación media sin previa coordinación.

Estas cuestiones son fundamentales para configurar "culturas del cuidado" de los estudiantes practicantes de profesorado. (LÓPEZ; SÁNCHEZ; ALTO- 
PIEDI, 2011). Promover acciones desde ambas instituciones involucradas que conlleven a un acompañamiento, contención y apoyo de los practicantes, es apuntar al cuidado de los noveles docentes. De tal manera, promover la cultura institucional del apoyo a los futuros docentes de educación media en esta primera etapa de formación profesional podría ser una respuesta para revertir las ya clásicas metáforas del aviador - "aterriza como puedas" - o la del nadador - "nada o ahógate" (MARCELO, 1999) que aplicadas al caso que nos preocupa podríamos adaptar como "aprende a nadar y dar clases en el centro educativo que te toque o consigas" o "aterriza en la institución de práctica como puedas".

La buena articulación y coordinación entre institución formadora e institución de práctica es una característica necesaria para que se pueda instalar lo que la UNESCO denomina "cultura innovadora". (OREALC-UNESCO, 2006, p. 29). La misma supone un conjunto de actitudes, necesidades y expectativas que deben ser conocidas y compartidas por toda la comunidad escolar (profesores, estudiantes, profesores practicantes, personal administrativo, etc.), para que el centro tenga una actitud positiva hacia los procesos de cambio, comprometido con la mejora de los procesos educativos.

Los vínculos institucionales que deben darse entre la institución formadora y la institución de práctica, implican importantes desafíos para construir proyectos y programas de común acuerdo. En tal sentido, los datos examinados contribuyen a reflexionar y pensar alternativas y programas de formación de profesores donde la Escuela de práctica se constituya en el eje central de la formación docente.

El diseño de un plan de formación integral basado en la práctica formalmente establecida mediante acuerdos inter institucionales entre escuelas de práctica que reciben estudiantes de profesorado y centros de formación inicial (institutos o Universidades) es un paso importante en el largo camino de fortalecimiento de la calidad en la formación docente.

\section{REFERENCIAS}

ALLIAUD, A. La experiencia escolar de maestros "inexpertos". Biografías, trayectorias y práctica profesional. Revista Iberoamericana de Educación, n. 34/3, 2006.

ANECA. Agencia Nacional de Evaluación de la Calidad y Acreditación. La dimensión práctica en la formación inicial del profesorado de secundaria. Ministerio de Educación, Política Social y Deporte; Agencia Nacional de Evaluación de la Calidad y Acreditación. Madrid, 2009. 
BAQUERO MÁSMELA, P. Prácticas pedagógicas, investigación y formación de educadores. Tres concepciones dominantes de la práctica docente. Revista Actualidades Pedagógicas, n. 49, p. 9-22, 2006.

BRASLAVSKY, C. La Educación Secundaria en el contexto de los cambios en los sistemas educativos latinoamericanos. Revista Iberoamericana de Educación, n. 9, p. 91-123, 1996.

CAMERON, R. The use of mixed methods in VET research. [El uso de métodos mixtos en la investigación en educación vocacional]. 2009. Disponible en: $<$ http://epubs.scu.edu. $\mathrm{au} /$ cgi/viewcontent.cgi? article $=1158 \&$ context $=$ comm_pubs $>$. Acceso en: 12 ago. 2016 .

CONTRERAS, I.; RITTERSHAUSSEN, S.; MONTECINOS, C.; SOLÍS, M.; NÚÑEZ, C.; WALKER, H. La Escuela como espacio para aprender a enseñar: Visiones desde los programas de Formación de Profesores de Educación Media. Estudios Pedagógicos, v. 36 , n. 1, p. 85-105, 2010.

CORREA MOLINA, E. La práctica docente: Una oportunidad de desarrollo profesional. Revista Perspectiva Educacional, v. 50, n. 2, p. 77-95, 2011.

CRESWELL, J.; PLANO, V. Designing and conducting mixed methods research. Thousand Oaks, CA: Sage, 2011.

DENZIN, N.; LINCOLN, Y. The Sage Handbook of Qualitative Research. Third Edition. Thousand Oaks: Sage Publications, 2012.

DIKER, G.; TERIGI, F. La formación de maestros y profesores: Hoja de ruta. Buenos Aires: Paidós, 1997.

DUBET, F. ¿Mutaciones institucionales y/o neoliberalismo? Revista Colombiana de Sociología, n. 25, p. 63-80, 2005.

ESCOBAR, N. La práctica profesional docente desde la perspectiva de los estudiantes practicantes y tutores. Revista Acción pedagógica, v. 16, n. 1, p. 182-193, 2007.

GRILLI, J.; SILVA, L. Análisis colectivo de las prácticas de aula. Dispositivos en la Formación Inicial de profesores que favorecen el aprendizaje colaborativo. Revista electrónica Diálogos Educativos, v. 15, n. 29, p. 69-89, 2015.

HERRÁN, A. El profesor que se forma. Desarrollo personal y profesional del docente. En: SÁNCHEZ HUETE, J. C. (Coord.). Compendio de Didáctica General. Madrid: CCS, 2008. p. 109-152.

IMBERNÓN, F. La formación y desarrollo profesional del profesorado. Hacia una nueva cultura profesional. Barcelona: Graó, 1994.

KADDOURI, M.; VANDROZ, D. Formation professionnelle en alternance: Quelques tensions d'ordre pédagogique et identitaire. En: CORREA MOLINA, E.; GERVAIS, C.; RITTERSHAUSSEN, S. (Dir.). Vers une conceptualisation de la situation de stage: Explorations internationales. Sherbrooke: Éditions du CRP, 2008. p. 23-42. 
KELLE, U. Sociological Explanations between Micro and Macro and the Integration of Qualitative and Quantitative Methods [43 paragraphs]. Forum Qualitative Sozialforschung/ Forum: Qualitative Social Research, v. 2, n. 1, Art. 5, 2001.

LESSARD, C.; BOURDONCLE, R. Les formations professionnelles universitaires. Place des praticiens et focalisation des savoirs pratiques: Utilité et limites. En: RAYMOND, D.; LENOIR, Y. (Dir.). Enseignants de métier et formation initiale: Des changements dans les rapports de formation à l'enseignement. Paris, Bruxelles: De Boeck Université, 1998. p. 11-33.

LINSTON, D.; ZEICHNER, K. Formación del profesorado y condiciones sociales de la escolarización. Madrid: Ediciones Morata, 2003.

LÓPEZ, J.; SÁNCHEZ, M.; ALTOPIEDI, M. Comunidades profesionales de práctica que logran sostener procesos de mejora institucional en las escuelas. Revista de Educación, n. 356, p. $109-131,2011$.

MARCELO, C. Estudio sobre estrategias de inserción profesional en Europa. Revista Iberoamericana de Educación, n. 19, p. 101-144, 1999.

MARCELO, C. El Profesor de Enseñanza Primaria. En: CONGRESO NACIONAL Y I IBEROAMERICANO DE PEDAGOGÍA, XII., 2000, Madrid. Anales... Madrid: Sociedad Española de Pedagogía, 26-30 de setiembre, 2000. Tomo I. Ponencias.

MARCELO, C.; ESTEVARRANZ, A. Modelos de colaboración entre la Universidad y las Escuelas de formación del profesorado. Revista de Educación, n. 317, p. 97-120, 1998.

MAXWELL, J. Qualitative research design. An Interactive Approach. Thousand Oaks, California: Sage Publications, 1996.

MECyT. Ministerio de Educación Superior, Ciencia y Tecnología. Lineamientos Curriculares Nacionales para la Formación Docente Inicial. Documento Aprobado. Resolución $\mathrm{n}^{\circ} 24 / 07.2014$. Disponible en: <file://C:/Documents\%20and\%20Settings/cseducacion/ Mis\%20documentos/Downloads/LINEAMIENTO\%20CURRICULAR\%20ARGENTINA.pdf>. Acceso en: 04 nov. 2015.

MINEDUC. Ministerio de Educación de Chile. Panel de Expertos para una Educación de Calidad. En: Propuestas para fortalecer la profesión docente en el sistema escolar chileno. Santiago: Ministerio de Educación, 9 Jul. 2010.

MONTECINOS, C.; WALKER, H.; CORTEZ, M.; MALDONADO, F. ¿Por qué y para qué los centros escolares aceptan ser un sitio de práctica para las carreras de pedagogía? Perspectivas de docentes directivos. Páginas de Educación, v. 6, n. 1, p. 37-59, 2013. Disponible en: <http://www.scielo.edu.uy/pdf/pe/v6n1/v6n1a03.pdf>. Acceso en: 12 ago. 2016.

OCDE. Organización para la Cooperación y el Desarrollo Económicos. Los docentes son importantes: Atraer, formar y conservar a los docentes eficientes. 2009. Publicado 
originalmente por la OCDE en inglés bajo el título: Teachers Matter: Attracting. Developing and Retaining Effective Teachers. (OECD, 2005).

OREALC-UNESCO. Modelos innovadores en la formación inicial docente. Estudio de casos de modelos innovadores en la formación docente en América Latina y Europa. UNESCO, Oficina Regional de Educación para América Latina y el Caribe. Chile: Andros Impresores, 2006.

PÉREZ GÓMEZ, Á. Practical Training and the Professional Socialization of Future Teachers in Andalucia. Prospects, UNESCO, n. 99, Geneva, 1996.

RODRÍGUEZ ZIDÁN, E.; GRILLI, J. Un estudio sobre la pareja pedagógica como dispositivo en la formación inicial de profesores para la educación media en Uruguay. Revista Electrónica Diálogos Educativos, v. 12, n. 23, p. 38-65, 2012.

RODRÍGUEZ ZIDÁN, E.; GRILLI, J. La pareja pedagógica: Una estrategia para transitar y aprender el oficio de ser profesor. Páginas de Educación, v. 6, n. 1, p. 54-73, 2013.

SANJURJO, L. Socializar experiencias de formación en prácticas profesionales: Un modo de desarrollo profesional. Revista Praxis Educativa, v. 16, n. 1, p. 22-32, 2012. Disponible en: <http://www.biblioteca.unlpam.edu.ar/pubpdf/praxis/v16n1a03sanjurjo. pdf $>$. Acceso en: 12 ago. 2016.

TALLAFERRO, D. La formación para la práctica reflexiva en las prácticas profesionales docentes. Revista Educere, v. 10, n. 33, p. 269-273, 2006.

TEJADA FERNÁNDEZ, J.; RUIZ BUENO, C. Significación del Prácticum en la asquisición de competencias profesionales que permiten la transferencia de conocimientos a ámbitos propios de la acción docente. Profesorado, Revista del currículum y formación del profesorado, v. 17, n. 3, p. 91-110, 2013.

TERIGI, F. Diez miradas sobre la escuela primaria. Buenos Aires: Fundación OSDE: Siglo XXI Editores, 2006.

TYACK, D.; CUBAN, L. En busca de la utopía. Un siglo de reformas de las escuelas públicas. 2. ed. México: Fondo de Cultura Económica, 2001.

URRUTIA, E. De la importancia de las Prácticas en la Formación Inicial Docente: Una aproximación desde la experiencia. Docencia, n. 43, p. 89-91, 2011.

VAILLANT, D.; MANSO, J. Tendencias en la formación inicial docente. Cuadernos de Investigación Educativa, v. 3, n. 18, p. 11-30, 2012.

VAILLANT, D.; MARCELO, C. Las tareas del Formador. Málaga: Ediciones Aljibre, 2001.

VAILLANT, D.; MARCELO, C. El ABC y D de la Formación Docente. Madrid: Narcea, 2015.

VAILLANT, D.; WETTSTEIN, G. (Ed.). Centros Regionales de Profesores una apuesta al Uruguay del siglo XX. Montevideo: Editorial Fin de Siglo, 2007. 
VEENMAN, S. Perceived problems of beginning teachers. Review of Educational Research, v. 54, n. 2, p. 143-178, 1984.

VEENMAN, S. El proceso de llegar a ser profesor: Un análisis de la formación inicial. En: VILLA, A. (Ed.). Perspectivas y problemas de la función docente. Madrid: Narcea, 1988.

VEZUB, L. La formación y el desarrollo profesional docente frente a los nuevos desafíos de la escolaridad. Profesorado. Revista de currículum y formación del profesorado, v. 11, n. 1, p. 1-23, 2007.

WIDEEN, M.; MAYER-SMITH, J.; MOON, B. A critical analysis of the research on learning to each: Making the case for an ecological perspective on inquiry. Review of Educational Research, v. 68, n. 2, p. 130-178, 1998.

ZANTING, A.; VERLOOP, N.; VERMUNT, J. Student teachers eliciting mentor's practical knowledge and comparing it to their own beliefs. Teaching and Teacher Education, n. 17 , p. 725-740, 2001.

ZEICHNER, K. Designing educative practicum experiences for prospective teachers. En: ZEICHNER, K.; MELNICK, S.; GOMEZ, M. (Dir.). Currents of Reform in Preservice Teacher Education. New York: Teachers College Press, 1996. p. 215-234.

Texto recibido en 19 de abril de 2016. Texto aprobado en 29 de junio de 2016. 


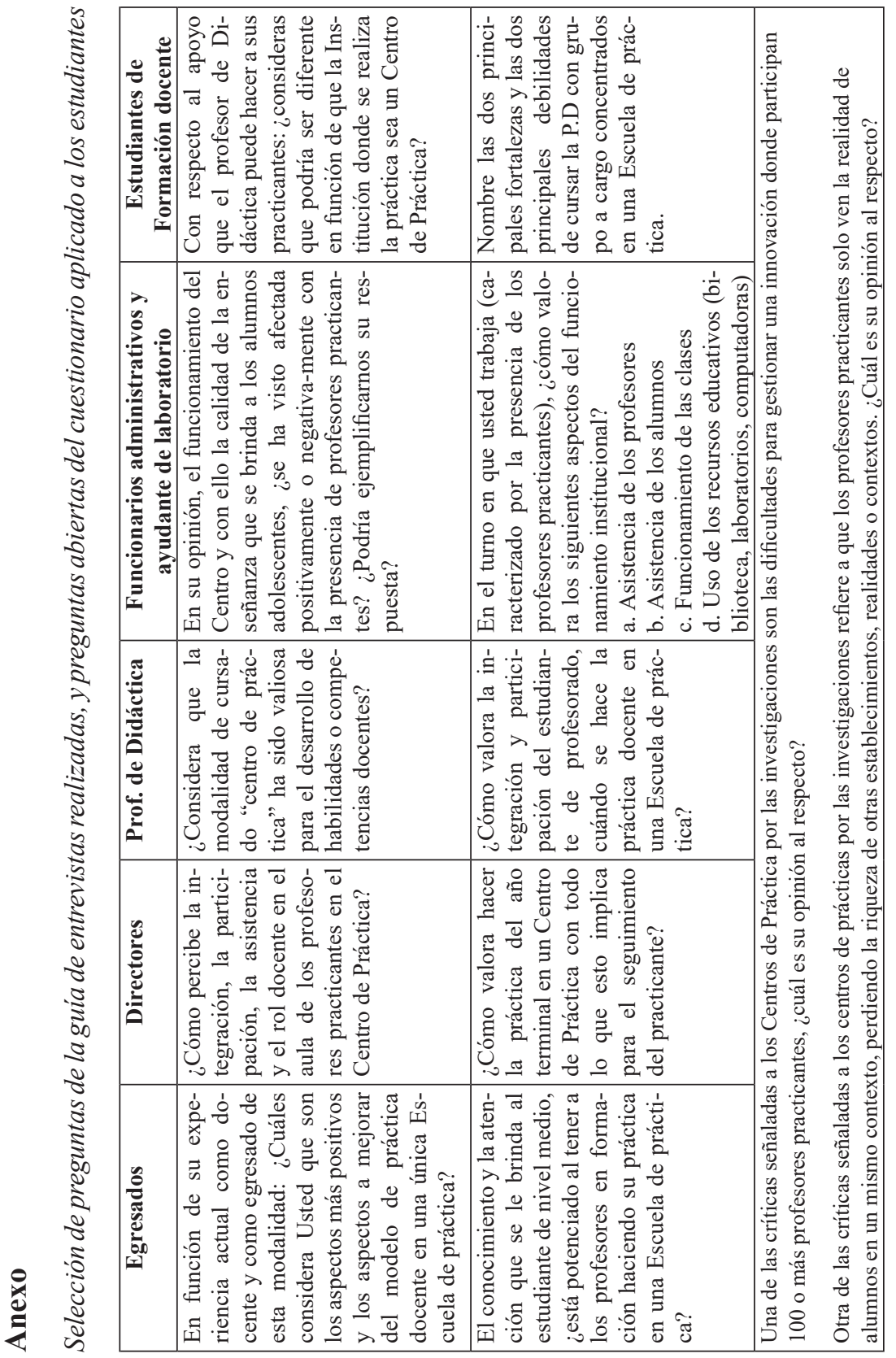


